\title{
The Enumerative Geometry of Hyperplane Arrangements
}

\author{
Thomas Paul, Will Traves and Max Wakefield*
}

\begin{abstract}
We study enumerative questions on the moduli space $\mathcal{M}(L)$ of hyperplane arrangements with a given intersection lattice $L$. Mnëv's universality theorem suggests that these moduli spaces can be arbitrarily complicated; indeed it is even difficult to compute the dimension $D=\operatorname{dim} \mathcal{M}(L)$. Embedding $\mathcal{M}(L)$ in a product of projective spaces, we study the degree $N=\operatorname{deg} \mathcal{M}(L)$, which can be interpreted as the number of arrangements in $\mathcal{M}(L)$ that pass through $D$ points in general position. For generic arrangements $N$ can be computed combinatorially and this number also appears in the study of the Chow variety of zero dimensional cycles. We compute $D$ and $N$ using Schubert calculus in the case where $L$ is the intersection lattice of the arrangement obtained by taking multiple cones over a generic arrangement. We also calculate the characteristic numbers for families of generic arrangements in $\mathbb{P}^{2}$ with 3 and 4 lines.
\end{abstract}

\section{Introduction}

Enumerative geometry has a monumental history and continues to be an inspiration for many different fields of research (for example see Katz [13] and Kontsevich and Manin [17]). Solutions to enumerative problems have given deep insight into the geometric nature of various algebraic varieties and important spaces.

Realization spaces of matroids (or moduli spaces of hyperplane arrangements) give a beautiful connection between combinatorics and algebraic geometry. In particular, Mnëv's universality theorem presents matroids as a kind of dictionary for quasi-projective varieties (see Mnëv [18] or Vakil [27]). These realization spaces have been studied from many different viewpoints. Kapranov [12] showed the Hilbert compactification of the moduli

*Department of Mathematics, U.S. Naval Academy, traves@usna.edu, wakefiel@usna.edu 
space of generic arrangements could be viewed as a Chow quotient. Hacking, Keel, and Tevelev [10] applied the relative minimal model program to the moduli space of arrangements. Terao [25] studied the closure of this moduli space in a product of projective spaces and an associated logarithmic Gauss-Manin connection. Speyer [24] proved that the $K$ theory class (actually a push forward-pullback) of the inclusion of the moduli space into the appropriate Grassmannian is actually the 2-variable Tutte polynomial of the associated matroid. Then Fink and Speyer [6] generalized this result to non-realizable matroids. In this note we study some of the geometry of this moduli space and find another use of the Tutte polynomial.

One of our motivating problems is Terao's conjecture which concerns the subset of the realization consisting of free arrangements (see Orlik and Terao [19] for a general reference on hyperplane arrangements). Yuzvinsky [28] showed that this subset was Zariski-open. It is not known if this subset is also closed. Our focus here is on computing the dimension and essentially the degree of this realization space by using both combinatorial and geometric methods.

To each hyperplane arrangement $\mathcal{A}=\left\{H_{1}, \ldots, H_{k}\right\}$ in $\mathbb{P}^{n}$ we associate an intersection lattice $\mathcal{L}(\mathcal{A})$, the poset whose elements are intersections $H_{i_{1}} \cap \cdots \cap H_{i_{t}}$ ordered by reverse inclusion, $B \leq C \Longleftrightarrow C \subseteq B$. Two arrangements $\mathcal{A}$ and $\mathcal{A}^{\prime}$ are combinatorially equivalent if their intersection lattices are isomorphic, that is, if there is a bijection $\phi$ : $\mathcal{A} \rightarrow \mathcal{A}^{\prime}$ that preserves the lattice order, $B \leq C \Longleftrightarrow \phi(B) \leq \phi(C)$. Let $\mathcal{M}_{\mathcal{A}}$ be the set of arrangements that are combinatorially equivalent to the arrangement $\mathcal{A}$. Identifying each arrangement $\left\{H_{1}, \ldots, H_{k}\right\}$ with its orbit $\left\{\left(H_{\sigma(1)}, \ldots, H_{\sigma(k)}\right): \sigma \in \mathcal{S}_{k}\right\}$ under the permutation group $\mathcal{S}_{k}$, we obtain an embedding $\mathcal{M}_{\mathcal{A}} \hookrightarrow\left(\mathbb{P}^{n *}\right)^{k} / \mathcal{S}_{k}$ and we give $\mathcal{M}_{\mathcal{A}}$ the induced topology coming from the Zariski topology on the quotient space. It is clear that $\mathcal{M}_{\mathcal{A}}$ depends only on the intersection lattice of $\mathcal{A}$. If $D=\operatorname{dim} \mathcal{M}_{\mathcal{A}}$ is the dimension of $\mathcal{M}_{\mathcal{A}}$, then let $N_{\mathcal{A}}$ be the number of arrangements in $\mathcal{M}_{A}$ passing through $D$ points in general position in $\mathbb{P}^{n}$.

Question 1. Given $\mathcal{A}$ compute $D$ and $N_{\mathcal{A}}$. Ideally these answers should be given in terms of the combinatorics of the lattice $\mathcal{L}(\mathcal{A})$.

In the next section we show that when $\mathcal{A}$ is a generic arrangement of $k$ hyperplanes in $\mathbb{P}^{n}$, then $\operatorname{dim} \mathcal{M}_{\mathcal{A}}=k n$ and we compute $N_{\mathcal{A}}$. The characteristic number $N_{\mathcal{A}}(p, \ell)$ measures the number of arrangements combinatorially equivalent to $\mathcal{A}$ that pass through $p$ points and are tangent to $\ell$ lines in general position (with $p+\ell=\operatorname{dim} \mathcal{M}_{\mathcal{A}}$ ). We use intersection theory on the correspondence between hyperplane arrangements and their dual arrangements to compute the characteristic numbers for generic arrangements of three or four lines in $\mathbb{P}^{2}$ (a good reference for the intersection theory that we use is Eisenbud and Harris [4] or Fulton [7]). This seems to be the first computation of these characteristic 
numbers for line arrangements though characteristic numbers were computed for smooth curves of degrees 3 and 4 by Zeuthen [29] in the $19^{\text {th }}$ century. As reported in Kleiman [15], the $19^{\text {th }}$ century methods lacked adequate foundations prompting Hilbert to ask for a rigorous computation of these characteristic numbers. Aluffi [1] and Kleiman and Speiser [16] verified Zeuthen's degree 3 predictions using intersection theory. Vakil [26] used intersection theory on the moduli space of stable maps to verify the degree 4 predictions. The importance of the characteristic numbers is suggested by a theorem originally due to Zeuthen [30] (also see Fulton [7, section 10.4]) that shows that the characteristic numbers for a family of curves determine the number of such curves tangent to smooth curves of arbitrary degrees. We close Section 2 by interpreting this theorem for line arrangements. It would be interesting to use intersection theory on the moduli space of stable maps to recover our results.

In Section 3 we consider cones over generic arrangements of hyperplanes.

Definition 2. A $d$-coned generic arrangement $\mathcal{A}$ in $\mathbb{P}^{n}$ is an arrangement of $k>n$ hyperplanes obtained from a generic hyperplane arrangement $\mathcal{B}$ in a linear subspace $H \cong$ $\mathbb{P}^{n-(d+1)}$ by taking the cone with a $d$-dimensional linear space (equivalently, with $d+1$ general points). That is, there exists a linear space $\Omega$ of dimension $d$, disjoint from $H$ so that each hyperplane in $\mathcal{A}$ is the linear span of both a hyperplane in $\mathcal{B}$ and $\Omega$.

In Section 3 we answer the enumerative problems from Question 1 for $d$-coned generic arrangements. Here the methods of Schubert Calculus come into play and the Catalan numbers make a cameo appearance.

Our approach to computing $N_{A}$ is to first compute the number of labeled arrangements with intersection lattice isomorphic to $\mathcal{L}(\mathcal{A})$ that pass through $D$ points in general position in $\mathbb{P}^{n}$. Dividing by the number of ways to label the hyperplanes in $\mathcal{A}$ gives $N_{A}$. This allows us to work in a product of polynomial rings rather than its quotient. A recent paper by Fehér, Némethi, and Rimányi [5] also studies enumerative problems involving hyperplane arrangements; they embrace quotient varieties and work with equivariant cohomology.

Though we were not able to answer Question 1 in terms of the combinatorics of $\mathcal{L}(\mathcal{A})$, we remain optimistic about this possibility for special families of arrangements despite several warning signs that the question may be very difficult in general. Mnëv's Universality Theorem says that each variety appears as the closure of $\mathcal{M}_{\mathcal{A}}$ for some hyperplane arrangement $\mathcal{A}$, so the geometry of $\mathcal{M}_{\mathcal{A}}$ can be arbitrarily complicated. Another warning sign appears if we take a naive approach to the dimension problem in $\mathbb{P}^{2}$. For each line arrangement $\mathcal{A}$ with $\ell$ labeled lines $L_{1}, \ldots, L_{\ell}$ and $k$ labeled points $p_{1}, \ldots, p_{k}$ of intersection among these lines, form the parameter space

$$
P_{\mathcal{A}}=\left\{\left(H_{1}, \ldots, H_{\ell}, P_{1}, \ldots, P_{k}\right) \in\left(\mathbb{P}^{2 *}\right)^{\ell} \times\left(\mathbb{P}^{2}\right)^{k}: P_{i} \in H_{j} \text { if } p_{i} \in L_{j}\right\} .
$$


Note that $\operatorname{dim} P_{\mathcal{A}}=\operatorname{dim} \mathcal{M}_{\mathcal{A}}$. If the conditions $P_{i} \in H_{j}$ are algebraically independent, then this dimension is $2(k+\ell)-\sum_{i=1}^{k}\left|\left\{L_{j}: p_{i} \in L_{j}\right\}\right|$ (sometimes this is called the virtual dimension of $\mathcal{M}_{\mathcal{A}}$ ). Such a formula holds for generic line arrangements and pencils as well as for many other arrangements; however, it fails for the Pappus arrangement pictured in Figure 1, since one of the conditions $P_{i} \in H_{j}$ is implied by the others. Indeed, the dimension of $\mathcal{M}_{\mathcal{A}}$ depends on the syzygies among these incidence conditions and so any potential algorithms need to be sensitive enough to recognize such syzygies from the combinatorial information in $\mathcal{L}(A)$, a task that appears to be quite difficult. This example (more precisely, its projective dual) was studied in detail by Fehér, Némethi, and Rimányi [5] and by Ren, Richter-Gebert and Sturmfels [21].

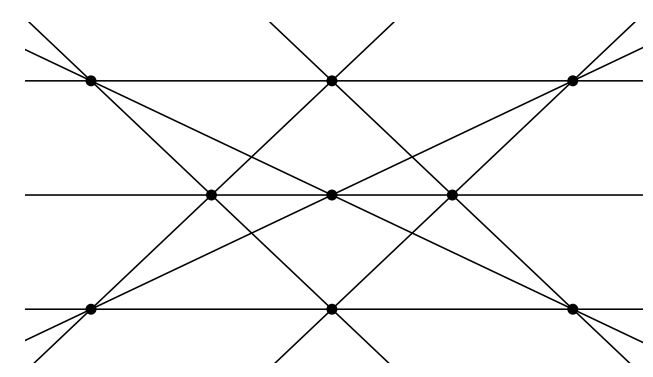

Figure 1: The Pappus arrangement consists of nine lines. The 27 point-line incidence conditions are dependent: the incidence correspondence has codimension 26.

Remark 3. We use the notation $\left\{(a)^{a^{\prime}},(b)^{b^{\prime}}, \ldots,(k)^{k^{\prime}}\right\}$ (sometimes omitting the braces) to refer to the multiset consisting of $a^{\prime}$ copies of $a, b^{\prime}$ copies of $b$, et cetera. Similarly, we use $\left(\underset{(a)^{a^{\prime}},(b)^{b^{\prime}}, \ldots,(k)^{k^{\prime}}}{ }\right)$ to refer to the multinomial that counts the ways of choosing $a^{\prime}$ groups of $a$ objects, $b^{\prime}$ groups of $b$ objects, ..., and $k^{\prime}$ groups of $k$ objects from $d$ labeled objects. That is, $\left(\underset{(a)^{a^{\prime}},(b)^{b^{\prime}}, \ldots,(k)^{k^{\prime}}}{d}\right)=d ! /(a !)^{a^{\prime}} \cdots(k !)^{k^{\prime}}$.

\section{Generic Arrangements}

An arrangement of $k$ hyperplanes in $\mathbb{P}^{n}$ is said to be generic if $k>n$ and no point is in the intersection of more than $n$ of the hyperplanes. Carlini discovered the following fact while studying the Chow variety of zero dimensional degree- $k$ cycles in $\mathbb{P}^{n}[3$, Proposition 3.4].

Theorem 4. When $\mathcal{A}$ is a generic arrangement of $k$ hyperplanes in $\mathbb{P}^{n}$ then the dimension $D$ of $\mathcal{M}_{\mathcal{A}}$ is $k n$ and the number of arrangements with lattice type isomorphic to $\mathcal{L}(A)$ that 
pass through D points in general position in $\mathbb{P}^{n}$ is

$$
N_{\mathcal{A}}=\frac{1}{k !}\left(\begin{array}{c}
k n \\
n
\end{array}\right)\left(\begin{array}{c}
(k-1) n \\
n
\end{array}\right) \cdots\left(\begin{array}{l}
n \\
n
\end{array}\right)=\frac{(k n) !}{k !(n !)^{k}} .
$$

Proof. The moduli space $\mathcal{M}_{\mathcal{A}}$ is a $k$ ! to 1 cover of the complement of a closed set in $\left(\mathbb{P}^{n *}\right)^{k}$ that parameterizes the sets of $k$ hyperplanes that contain a set of $n$ linearly dependent hyperplanes. So $D=\operatorname{dim} \mathcal{M}_{\mathcal{A}}=\operatorname{dim}\left(\mathbb{P}^{n *}\right)^{k}=k n$. We count the ordered (or labeled) generic arrangements passing through $k n$ points in general position. Since the points are in general position, no more than $n$ can lie on any one hyperplane. So by the pigeonhole principle, each hyperplane contains precisely $n$ of the points and each hyperplane is completely determined by these $n$ points. There are clearly $\prod_{i=0}^{k-1}\left(\begin{array}{c}k n-i n \\ n\end{array}\right)$ ways to distribute the points among the labeled hyperplanes, but each hyperplane arrangement can be labeled in $k$ ! ways so dividing the product of binomial coefficients by $k$ ! gives $N_{\mathcal{A}}$.

Remark 5. We remark that when $n=2$ the formula for $N_{\mathcal{A}}$ reduces to $(2 k-1)$ !!. Aside from the nice simplicity of this result, this formulation allows us to interpret the result in terms of the multivariate Tutte polynomial of the lattice $\mathcal{L}(\mathcal{A})$. The multivariate Tutte polynomial of $\mathcal{L}(\mathcal{A})$ (see Ardila [2] or Sokal [23]) is defined as

$$
Z_{\mathcal{L}(\mathcal{A})}\left(q, x_{1}, \ldots, x_{k}\right)=\sum_{B \in \mathcal{L}(\mathcal{A})}\left(q^{-r k(B)}\right) \prod_{H_{j} \in B} x_{j},
$$

where if $B \subset \mathcal{A}$ then $r k(B)=\operatorname{codim} \cap_{H \in B} H$. When $\mathcal{A}$ is a generic arrangement with $k$ hyperplanes,

$$
Z_{\mathcal{L}(\mathcal{A})}\left(1, x_{1}, \ldots, x_{k}\right)=\sum_{B \subset \mathcal{A}} \prod_{H_{j} \in B} x_{j}=\left(1+x_{1}\right)\left(1+x_{2}\right) \cdots\left(1+x_{k}\right) .
$$

In particular,

$$
Z_{\mathcal{L}(\mathcal{A})}(1,0,2, \ldots, 2 k-2)=(2 k-1) ! !=N_{\mathcal{A}} .
$$

Remark 6. The intersection ring (or Chow ring) $A(X)$ of a variety $X$ can be defined as the ring of equivalence classes of algebraic subvarieties of $X$ modulo rational equivalence, graded by codimension [7]. Here $\left[Y_{1} \cup Y_{2}\right]=\left[Y_{1}\right]+\left[Y_{2}\right]$. Also, $\left[Y_{1} \cap Y_{2}\right]=\left[Y_{1}\right] \cdot\left[Y_{2}\right]$ if $Y_{1}$ and $Y_{2}$ intersect transversely. The intersection rings that we consider can also be interpreted in terms of cohomology; for more on this point of view, see Katz [13].

When $X \cong \mathbb{P}^{n_{1}} \times \cdots \times \mathbb{P}^{n_{k}}$ is a product of projective spaces then

$$
A(X) \cong \frac{\mathbb{Z}\left[x_{1}, \ldots, x_{k}\right]}{\left(x_{1}^{n_{1}+1}, \ldots, x_{k}^{n_{k}+1}\right)}
$$

where the $x_{i}$ are the pullbacks of the classes of hyperplanes on each factor to the product. 
Next we prove a Lemma that will be used in many of the following arguments.

Lemma 7. Fix an arrangement $\mathcal{A}$ in $\mathbb{P}^{n}$ with $D=\operatorname{dim} \mathcal{M}_{\mathcal{A}}$ and $|\mathcal{A}|=k$. The conditions that the arrangement contain $D$ specified points in general position are transverse and the class corresponding to the intersection of these conditions is

$$
\left(\sum_{i=1}^{k} x_{i}\right)^{D}
$$

Proof. The class of the condition that a point lies on the $i$ th hyperplane is just $x_{i}$. Then the class of the condition that a point lies on one of the $k$ hyperplanes is $\sum_{i=1}^{k} x_{i}$. Now, the action of $\operatorname{PGL}(n)^{k}$ is transitive on $\mathcal{M}_{\mathcal{A}}$. Since the $D$ point conditions are assumed to be generic we can use this $\operatorname{PGL}(n)^{k}$ action to move one point condition to another. Hence Kleiman's Transversality Theorem [14] says that these conditions are transverse and the corresponding Chow class is the product of the classes.

Note that Theorem 4 could also be easily proved by Lemma 7. Since we are not putting any conditions on the generic lines we have nothing but point conditions. Then the degree of the Chow class given in Lemma 4 counts the number of ordered generic hyperplane arrangements passing through $D$ points in general position. Dividing by $k$ ! gives the number of such unordered generic arrangements.

Continuing to focus on the case where $n=2$, we will compute the characteristic numbers of generic line arrangements with $k=3$ or $k=4$ lines. We define the characteristic number $N_{\mathcal{A}}(p, \ell)$ to be the number of arrangements with the same lattice type as $\mathcal{A}$ that pass through $p$ points and are tangent to $\ell$ lines in general position (with $p+\ell=\operatorname{dim} \mathcal{M}_{\mathcal{A}}$ ). When $\mathcal{A}$ is a generic arrangement of $k$ lines, we simplify the notation to $N_{k}(p, \ell)$. To get these numbers we compute the degree of a related variety $M$ which is a delicate intersection calculation. We do this in two different ways. First for the case $k=3$ we preform an explicit calculation using the method of undetermined coefficients. Then for the case $k=4$ we argue that the corresponding intersection class is a product using transversality considerations.

Remark 8. The only way that an arrangement of 3 lines $\mathcal{A}=\left\{\ell_{1}, \ell_{2}, \ell_{3}\right\}$ can be tangent to a given line $L$ is if the cubic defining the arrangement meets the line $L$ at a point of multiplicity strictly greater than 1 . If the arrangement is generic this means that an intersection point $\ell_{i} \cap \ell_{j}$ of two of the 3 lines must lie on $L$. This motivates the introduction of the points $p_{i j}=\ell_{i} \cap \ell_{j}$ in the following lemma: a generic arrangement is tangent to a line $L$ if and only if there exists a point $p_{i j}$ on $L$. 
Lemma 9. The set

$$
M=\left\{\left(\ell_{1}, \ell_{2}, \ell_{3}, p_{12}, p_{13}, p_{23}\right) \in\left(\mathbb{P}^{2 *}\right)^{3} \times\left(\mathbb{P}^{2}\right)^{3}: p_{i j}=\ell_{i} \cap \ell_{j} \text { for } 1 \leq i<j \leq 3\right\}
$$

is a quasi-projective variety of dimension 6 in $\left(\mathbb{P}^{2 *}\right)^{3} \times\left(\mathbb{P}^{2}\right)^{3}$. The Chow ring is

$$
A=A\left(\left(\mathbb{P}^{2 *}\right)^{3} \times\left(\mathbb{P}^{2}\right)^{3}\right) \cong \mathbb{Z}\left[x_{1}, x_{2}, x_{3}, y_{12}, y_{13}, y_{23}\right] /\left(x_{1}^{3}, x_{2}^{3}, x_{3}^{3}, y_{12}^{3}, y_{13}^{3}, y_{23}^{3}\right)
$$

where the $x_{i}$ are the classes of the lines and the $y_{i j}$ are the classes of the points. The class of $M$ in $A$ is

$$
[M]=\prod_{1 \leq i<j \leq 3}\left(x_{i}+y_{i j}\right)\left(x_{j}+y_{i j}\right) .
$$

Proof. Since $M$ has codimension 6 the class $[M]$ is a degree 6 polynomial in the Chow ring $A$. We write this class as the sum of all degree 6 monomials in $A$ :

$$
[M]=\sum_{a_{1}+\cdots+a_{6}=6} c_{\left(a_{i}\right)} x_{1}^{a_{1}} x_{2}^{a_{2}} x_{3}^{a_{3}} y_{12}^{a_{4}} y_{13}^{a_{5}} y_{23}^{a_{6}}
$$

Now we determine all the coefficients. First we show which coefficients are zero. To do this we first partition the set of degree- 6 monomials that appear in the expanded product into types. In the definitions that follow the notation $p \in A_{i} / I$ denotes a degree- $i$ monomial in the variables appearing in the quotient ring $A / I$.

The first type of monomials we define are

$$
\begin{gathered}
T_{1}=\left\{\left\{x_{1}^{2} y_{12}^{2} p \mid p \in A_{2} /\left(x_{1}, y_{12}\right)\right\} \bigcup\left\{x_{2}^{2} y_{12}^{2} p \mid p \in A_{2} /\left(x_{2}, y_{12}\right)\right\}\right. \\
\bigcup\left\{x_{1}^{2} y_{13}^{2} p \mid p \in A_{2} /\left(x_{1}, y_{13}\right)\right\} \bigcup\left\{x_{3}^{2} y_{13}^{2} p \mid p \in A_{2} /\left(x_{3}, y_{13}\right)\right\} \\
\left.\bigcup\left\{x_{2}^{2} y_{23}^{2} p \mid p \in A_{2} /\left(x_{2}, y_{23}\right)\right\} \bigcup\left\{x_{3}^{2} y_{23}^{2} p \mid p \in A_{2} /\left(x_{3}, y_{23}\right)\right\}\right\} .
\end{gathered}
$$

The second type we define are

$$
\begin{gathered}
T_{2}=\left\{\left\{x_{1}^{2} x_{2}^{2} y_{12} p \mid p \in A_{1} /\left(x_{1}, x_{2}, y_{12}\right)\right\} \bigcup\left\{x_{1}^{2} x_{3}^{2} y_{13} p \mid p \in A_{1} /\left(x_{1}, x_{3}, y_{13}\right)\right\}\right. \\
\left.\bigcup\left\{x_{2}^{2} x_{3}^{2} y_{23} p \mid p \in A_{1} /\left(x_{2}, x_{3}, y_{23}\right)\right\}\right\} .
\end{gathered}
$$

The third type we define are

$$
T_{3}=\left\{\left\{x_{1} y_{12}^{2} y_{13}^{2} p \mid p \in A_{1} /\left(x_{1}, y_{12}, y_{13}\right)\right\} \bigcup\left\{x_{2} y_{12}^{2} y_{23}^{2} p \mid p \in A_{1} /\left(x_{2}, y_{12}, y_{23}\right)\right\}\right.
$$




$$
\left.\bigcup\left\{x_{3} y_{23}^{2} y_{13}^{2} p \mid p \in A_{1} /\left(x_{3}, y_{23}, y_{13}\right)\right\}\right\} .
$$

The fourth type we define is

$$
T_{4}=\left\{x_{1}^{2} y_{23}^{2} x_{2} y_{12}, x_{1}^{2} y_{23}^{2} x_{3} y_{13}, x_{2}^{2} y_{13}^{2} x_{1} y_{12}, x_{2}^{2} y_{13}^{2} x_{3} y_{23}, x_{3}^{2} y_{12}^{2} x_{1} y_{13}, x_{3}^{2} y_{12}^{2} x_{2} y_{23}\right\} .
$$

Note that $\left|T_{1}\right|=54,\left|T_{2}\right|=9,\left|T_{3}\right|=9$, and $\left|T_{4}\right|=6$.

For any monomial $m \in A_{6}$ we define the complement of $m$ by the unique monomial $c(m)=m^{\prime}$ such that $m m^{\prime} \neq 0 \in A_{12}$. Set $Z_{1}=T_{1} \cup T_{2} \cup T_{3} \cup T_{4}$. Notice that $Z_{1}$ has the property that $c\left(Z_{1}\right)=Z_{1}$. Hence to show that the monomials in $Z_{1}$ have the coefficient of zero in $[M]$ we just check that multiplying $[M]$ by each of the monomials in $Z_{1}$ gives zero. Also, notice that within each type there is a union of smaller sets where each of those sets can be obtained from one another by a permutation of the coordinates. Hence if we can show that the coefficient is zero for one of the subsets then we have completed the task for the entire type. In the following arguments we use duality on $\mathbb{P}^{2}$ often.

First we argue $[M] m=0$ where $m \in T_{1}$. Let $m \in T_{1}$ be in the first subset so $m=x_{1}^{2} y_{12}^{2} m^{\prime}$. Then in $A$ this monomial $m$ represents fixing a generic line for $\ell_{1}$ in the first factor of $\mathbb{P}^{2 *}$ and a generic point for $p_{12}$. Set $\pi_{i}:\left(\mathbb{P}^{2 *}\right)^{3} \times\left(\mathbb{P}^{2}\right)^{3} \rightarrow \mathbb{P}^{2 *}$ to be the projection to the factor corresponding to the line $\ell_{i}$ and $\pi_{i j}:\left(\mathbb{P}^{2 *}\right)^{3} \times\left(\mathbb{P}^{2}\right)^{3} \rightarrow \mathbb{P}^{2}$ to be the projection to the factor corresponding to the point $p_{i j}$. With this notion we can say $m=\left[\pi_{1}^{-1}\right.$ (generic point $) \cap \pi_{12}^{-1}$ (generic point $) \cap\left(m^{\prime}\right.$ conditions $\left.)\right]$ where we will not need the conditions from $m^{\prime}$. Using the Moving Lemma (see [4, Theorem 5.4])

$$
\left.\left.[M] m=\left[M \cap \pi_{1}^{-1} \text { (generic point }\right) \cap \pi_{12}^{-1} \text { (generic point }\right) \cap\left(m^{\prime} \text { conditions }\right)\right] .
$$

In $M$ the point $p_{12}$ must lie on $\ell_{1}$ and this cannot happen if $\ell_{1}$ and $p_{12}$ are generic. Hence $[M] m=0$.

The other types have similar arguments. We include them for completeness, but with briefer arguments. For type 2 we look again at the first subset and put $m=x_{1}^{2} x_{2}^{2} y_{12} m^{\prime}$. Then $[M] m$ equals

$$
\left.\left.\left.\left[M \cap \pi_{1}^{-1} \text { (generic point }\right) \cap \pi_{2}^{-1} \text { (generic point }\right) \cap \pi_{12}^{-1} \text { (generic line }\right) \cap\left(m^{\prime} \text { conditions }\right)\right] .
$$

This means that we have fixed generic lines for lines 1 and 2 . Hence the point $p_{12}$ is fixed using $M$, but $p_{12}$ must also lie on a fixed line. We cannot find such a configuration; hence $[M] m=0$.

For type 3 we again look at the first subset $m=x_{1} y_{12}^{2} y_{13}^{2} m^{\prime}$. Then $[M] m$ equals

$$
\left.\left.\left[M \cap \pi_{1}^{-1} \text { (generic line) } \cap \pi_{12}^{-1} \text { (generic point }\right) \cap \pi_{13}^{-1} \text { (generic point }\right) \cap\left(m^{\prime} \text { conditions }\right)\right] .
$$


Line $\ell_{1}$ must satisfy the condition coming from $x_{1}$, which can be interpreted as saying that line $\ell_{1}$ lies in a fixed pencil of lines; however, this is inconsistent with the conditions requiring points $p_{12}$ and $p_{13}$ to lie in fixed (and general) positions. Hence $[M] m=0$.

For type 4 we just look at the first monomial $m=x_{1}^{2} y_{23}^{2} x_{2} y_{12}$. Then $[M] m$ equals

$\left[M \cap \pi_{1}^{-1}\right.$ (generic point) $\cap \pi_{23}^{-1}$ (generic point) $\cap \pi_{2}^{-1}$ (generic line) $\cap \pi_{12}^{-1}$ (generic line) $]$.

In this case $\ell_{1}$ must be a fixed generic line and $p_{23}$ must be a fixed generic point. Since line $\ell_{2}$ must lie in a fixed pencil and since $p_{23} \in \ell_{2}$, the line $\ell_{2}$ is fixed too. Then $p_{12}=\ell_{1} \cap \ell_{2}$ is fixed and so it cannot satisfy the generic linear condition in the class $[M] m$. Hence $[M] m=0$.

In this case we have fixed a generic line for line 1 and force $p_{23}$ to be fixed point in general position. The condition corresponding to $\left[\pi_{2}^{-1}\right.$ (generic line) $]$ forces line 2 to lie in a fixed pencil. Then line 2 is determined (since it passes through $p_{23}$ ) and so is $p_{12}=\ell_{1} \cap \ell_{2}$. However, the condition corresponding to $\left[\pi_{12}^{-1}\right.$ (generic line) $]$ forces $p_{12}$ to lie on a fixed general line (that is, not through $\ell_{1} \cap \ell_{2}$ ). Thus, we cannot find such a configuration and so $[M] m=0$.

Now we define another set of monomials and prove that each of these monomials have coefficient equal to 1 in $[M]$. Again we define this set as a union of a few different types. Unfortunately, there are many more types in this set even though there are less total monomials. We define the types in the following table.

\begin{tabular}{|c|c|}
\hline Type & Monomials \\
\hline$S_{1}$ & $\left\{x_{1}^{2} x_{2}^{2} x_{3}^{2}\right\}$ \\
\hline$S_{2}$ & $\left\{x_{1}^{2} x_{2}^{2} x_{3} y_{13}, x_{1}^{2} x_{2}^{2} x_{3} y_{23}, x_{1}^{2} x_{3}^{2} x_{2} y_{23}, x_{1}^{2} x_{3}^{2} x_{2} y_{12}, x_{2}^{2} x_{3}^{2} x_{1} y_{13}, x_{2}^{2} x_{3}^{2} x_{1} y_{12}\right\}$ \\
\hline$S_{3}$ & $\left\{x_{1}^{2} x_{2}^{2} y_{13} y_{23}, x_{1}^{2} x_{3}^{2} y_{12} y_{23}, x_{2}^{2} x_{3}^{2} y_{12} y_{13}\right\}$ \\
\hline$S_{4}$ & $\left\{x_{1}^{2} x_{2} x_{3} y_{23}^{2}, x_{2}^{2} x_{1} x_{3} y_{13}^{2}, x_{3}^{2} x_{2} x_{1} y_{12}^{2}\right\}$ \\
\hline$S_{5}$ & $\left\{x_{1}^{2} x_{2} x_{3} y_{12} y_{13}, x_{2}^{2} x_{1} x_{3} y_{12} y_{23}, x_{3}^{2} x_{1} x_{2} y_{13} y_{23}\right\}$ \\
\hline $\bar{S} S_{6}$ & $\begin{array}{l}\left\{x_{1}^{2} x_{2} x_{3} y_{23} y_{12}, x_{1}^{2} x_{2} x_{3} y_{23} y_{13}, x_{2}^{2} x_{1} x_{3} y_{13} y_{12}, x_{2}^{2} x_{1} x_{3} y_{13} y_{23},\right. \\
\left.x_{3}^{2} x_{2} x_{1} y_{12} y_{13}, x_{3}^{2} x_{2} x_{1} y_{12} y_{23}\right\}\end{array}$ \\
\hline$S_{7}$ & $\left\{x_{1}^{2} x_{2} y_{13} y_{23}^{2}, x_{1}^{2} x_{3} y_{12} y_{23}^{2}, x_{2}^{2} x_{1} y_{23} y_{13}^{2}, x_{2}^{2} x_{1} y_{23} y_{13}^{2}, x_{3}^{2} x_{1} y_{23} y_{12}^{2}, x_{3}^{2} x_{2} y_{13} y_{12}^{2}\right\}$ \\
\hline$S_{8}$ & $\begin{array}{l}\left\{x_{1}^{2} x_{2} y_{12} y_{13} y_{23}, x_{1}^{2} x_{3} y_{12} y_{13} y_{23}, x_{2}^{2} x_{1} y_{12} y_{13} y_{23}, x_{2}^{2} x_{3} y_{12} y_{13} y_{23},\right. \\
\left.x_{3}^{2} x_{2} y_{12} y_{13} y_{23}, x_{3}^{2} x_{1} y_{12} y_{13} y_{23}\right\}\end{array}$ \\
\hline$S_{9}$ & $\left\{x_{1}^{2} y_{23}^{2} y_{12} y_{13}, x_{2}^{2} y_{13}^{2} y_{23} y_{12}, x_{3}^{2} y_{12}^{2} y_{23} y_{13}\right\}$ \\
\hline$S_{10}$ & $\begin{array}{l}\left\{x_{1} x_{2} x_{3} y_{12}^{2} y_{13}, x_{1} x_{2} x_{3} y_{12}^{2} y_{23}, x_{1} x_{2} x_{3} y_{13}^{2} y_{12}, x_{1} x_{2} x_{3} y_{13}^{2} y_{23},\right. \\
\left.x_{1} x_{2} x_{3} y_{23}^{2} y_{12}, x_{1} x_{2} x_{3} y_{23}^{2} y_{13}\right\}\end{array}$ \\
\hline$S_{11}$ & $\left\{x_{1} x_{2} y_{12}^{2} y_{13} y_{23}, x_{1} x_{3} y_{12} y_{13}^{2} y_{23}, x_{3} x_{2} y_{12} y_{13} y_{23}^{2}\right\}$ \\
\hline$S_{12}$ & $\left\{x_{1} x_{2} y_{12} y_{13}^{2} y_{23}, x_{1} x_{2} y_{12} y_{13} y_{23}^{2}, x_{1} x_{3} y_{12}^{2} y_{13} y_{23}, x_{1} x_{3} y_{12} y_{13} y_{23}^{2}\right.$, \\
\hline
\end{tabular}




\begin{tabular}{|l|l|} 
& $\left.x_{3} x_{2} y_{12} y_{13}^{2} y_{23}, x_{3} x_{2} y_{12}^{2} y_{13} y_{23}\right\}$ \\
\hline$S_{13}$ & $\left\{x_{1} x_{2} y_{13}^{2} y_{23}^{2}, x_{1} x_{3} y_{12}^{2} y_{23}^{2}, x_{2} x_{3} y_{12}^{2} y_{13}^{2}\right\}$ \\
\hline$S_{14}$ & $\left\{x_{1} y_{12}^{2} y_{13} y_{23}^{2}, x_{1} y_{12} y_{13}^{2} y_{23}^{2}, x_{2} y_{12}^{2} y_{13}^{2} y_{23}, x_{2} y_{12} y_{13}^{2} y_{23}^{2}\right.$, \\
& $\left.x_{3} y_{12}^{2} y_{13} y_{23}^{2}, x_{3} y_{12}^{2} y_{13}^{2} y_{23}\right\}$ \\
\hline$S_{15}$ & $\left\{y_{12}^{2} y_{13}^{2} y_{23}^{2}\right\}$ \\
\hline
\end{tabular}

Now put $Z_{2}=S_{1} \cup \cdots \cup S_{15}$. In the next table we compute the intersection of $M \cap m$ where $m \in Z_{2}$. We briefly describe, for each type, how this intersection results in a unique arrangement of three lines with the marked points $p_{i j}$. Again we use the argument that within each $S_{i}$ we only need to check one monomial since all the others within that class can be obtained from permuting coordinates.

\begin{tabular}{|c|c|c|}
\hline Type & Monomial & Intersect this class with $M$ gives a unique point \\
\hline$\overline{S_{1}}$ & $x_{1}^{2} x_{2}^{2} x_{3}^{2}$ & Fixing three lines fixes the three intersection points. \\
\hline$S_{2}$ & $x_{1}^{2} x_{2}^{2} x_{3} y_{13}$ & $\begin{array}{l}\text { Fixing lines } 1 \text { and } 2 \text {. Then fixing a linear condition } \\
\text { point } 13 \text { gives a unique point and then putting } \\
\text { a linear condition on line } 3 \text { fixes it. }\end{array}$ \\
\hline$S_{3}$ & $x_{1}^{2} x_{2}^{2} y_{13} y_{23}$ & $\begin{array}{l}\text { Fixing lines } 1 \text { and } 2 \text {. Then giving linear conditions on points } \\
13 \text { and } 23 \text { fixes those points which fixes line } 3 \text {. }\end{array}$ \\
\hline$S_{4}$ & $x_{1}^{2} x_{2} x_{3} y_{23}^{2}$ & $\begin{array}{l}\text { Fix line } 1 \text { and point } 23 \text {. Then putting linear conditions on lines } \\
2 \text { and } 3 \text { fixes them. }\end{array}$ \\
\hline$S_{5}$ & $x_{1}^{2} x_{2} x_{3} y_{12} y_{13}$ & $\begin{array}{l}\text { Fix line } 1 . \text { Then Putting a linear condition on points } \\
12 \text { and } 13 \text { fixes them on line } 1 . \\
\text { Then putting linear conditions on lines } 2 \text { and } 3 \text { fixes them. }\end{array}$ \\
\hline$S_{6}$ & $x_{1}^{2} x_{2} x_{3} y_{12} y_{23}$ & $\begin{array}{l}\text { Fixing line } 1 \text { and giving a linear condition on point } 12 \\
\text { fixes point } 12 \text {. Together with putting a linear condition on } \\
\text { line } 2 \text { fixes it. Then putting a linear condition on point } 23 \\
\text { fixes it and line } 3 \text {. }\end{array}$ \\
\hline$S_{7}$ & $x_{1}^{2} x_{2} y_{13} y_{23}^{2}$ & $\begin{array}{l}\text { Fixing line } 1 \text {, point } 23 \text {, and putting a linear condition on } \\
\text { line } 2 \text { fixes it. Then putting a linear condition on point } 13 \\
\text { fixes it on line one and hence fixes line } 3 \text {. }\end{array}$ \\
\hline$S_{8}$ & $x_{1}^{2} x_{2} y_{12} y_{13} y_{23}$ & $\begin{array}{l}\text { Fix line } 1 \text { and putting liner conditions on points } \\
12 \text { and } 13 \text { fixes these points. Then putting a linear } \\
\text { condition on line } 2 \text { fixes it. And then finally fixing a linear } \\
\text { condition on point } 23 \text { fixes it and line } 3 \text {. }\end{array}$ \\
\hline$S_{9}$ & $x_{1} y_{23}^{2} y_{12} y_{13}$ & $\begin{array}{l}\text { Fix line } 1 \text { and point } 23 \text {. Putting linear conditions } \\
\text { on points } 12 \text { and } 13 \text { fixes them on line } 1 \text {. Then this } \\
\text { fixes lines } 2 \text { and } 3 .\end{array}$ \\
\hline
\end{tabular}




\begin{tabular}{|c|c|c|}
\hline$S_{10}$ & $x_{1} x_{2} x_{3} y_{12}^{2} y_{13}$ & $\begin{array}{l}\text { Fixing point } 12 \text { with putting linear conditions } \\
\text { on lines } 1 \text { and } 2 \text { fixes them. Then putting a linear condition on } \\
\text { point } 13 \text { fixes it on line } 1 \text {. Then putting a linear condition } \\
\text { on line } 3 \text { fixes it. }\end{array}$ \\
\hline$S_{11}$ & ${ }_{12}^{2} y_{13} y_{23}$ & $\begin{array}{l}\text { Fixing point } 12 \text { with putting linear conditions } \\
\text { on lines } 1 \text { and } 2 \text { fixes them. Then putting linear conditions on } \\
\text { points } 13 \text { and } 23 \text { fixes them and hence line } 3 \text {. }\end{array}$ \\
\hline$S_{12}$ & ${ }_{2} y_{13}^{2} y_{23}$ & $\begin{array}{l}\text { Fixing point } 13 \text { and putting a linear condition on } \\
\text { line } 1 \text { fixes it. Then putting a linear condition on point } 12 \text { fixes } \\
\text { it on line } 1 . \text { Then the linear condition on line } 2 \text { fixes it. } \\
\text { Then the linear condition on point } 23 \text { fixes it and line } 3 \text {. }\end{array}$ \\
\hline$S_{13}$ & ${ }_{2} y_{13}^{2} y_{23}^{2}$ & $\begin{array}{l}\text { Fixing points } 13 \text { and } 23 \text { fixes line } 3 \text {. Then putting lin } \\
\text { conditions on lines } 1 \text { and } 2 \text { fixes them }\end{array}$ \\
\hline$S_{14}$ & $x_{1} y_{12}^{2} y_{13} y_{23}^{2}$ & $\begin{array}{l}\text { Fixing points } 12 \text { and } 23 \text { fixes line } 2 \text {. Then giving a linear } \\
\text { condition on line } 1 \text { fixes it. Then putting a linear condition } \\
\text { on point } 13 \text { fixes it on line } 1 \text { which also fixes line } 3 \text {. }\end{array}$ \\
\hline$S_{15}$ & $y_{12}^{2} y_{13}^{2} y_{23}^{2}$ & The points 12,13 , and 23 are fixed. This fixes lines 1,2 , and 3. \\
\hline
\end{tabular}

Again since the complement $c\left(Z_{2}\right)=Z_{2}$ we have shown that the coefficients of all these monomials in $[M]$ are equal to 1 . There is just one more monomial to consider. Let $Z_{3}=\left\{x_{1} x_{2} x_{3} y_{12} y_{13} y_{23}\right\}$. This set also has the property $c\left(Z_{3}\right)=Z_{3}$. Now we compute its coefficient. The class $x_{1} x_{2} x_{3} y_{12} y_{13} y_{23}$ puts a linear condition on each line and point. For the lines we can think of this as fixing three points $q_{1}, q_{2}$, and $q_{3}$ in general position and requiring that $p_{i} \in \ell_{i}$, for $i \in\{1,2,3\}$. Similarly, to interpret the linear conditions on the points $p_{i j}$ we fix three lines $L_{12}, L_{13}$, and $L_{23}$ in general position, and require that $p_{i j} \in L_{i j}$.

Suppose now that we choose an arbitrary point $p_{12}$ on line $L_{12}$. Fix a embedding $\phi$ : $\mathbb{P}^{1} \rightarrow \mathbb{P}^{2}$ defined by $\phi([a: b])=\left[f_{1}(a: b): f_{2}(a: b): f_{3}(a: b)\right]$ where $\phi\left(\mathbb{P}^{1}\right)=L_{12}$ and each $f_{i}$ is homogeneous of degree 1 . Then there exists $[a: b] \in \mathbb{P}^{1}$ such that $\phi([a: b])=$ $p_{12}$. Now since the points $q_{1}$ and $p_{12}$ are fixed we have that line $1, \ell_{1}$, is fixed. Line $\ell_{1}$ fixed means that we can get $p_{13}=\ell_{1} \cap L_{13}$. Next we find $\ell_{3}$ is determined by the points $p_{13}$ and $q_{3}$. With $\ell_{3}$ we can get $p_{23}=\ell_{3} \cap L_{23}$. Now we make $\ell_{2}$ the line through the points $p_{23}$ and $q_{2}$. Since we chose $p_{12}$ randomly at the beginning of this process we have that $P=\ell_{2} \cap L_{12}$ may not be equal to $p_{12}$. Each step in the above process to determine each line and point is a cross product computation with a vector of constants (the line or point that is fixed before the choice of $p_{12}$ ) with a vector of homogenous degree 1 in the functions $f_{i}$. Hence there exist linear functions $g_{1}$ and $g_{2}$ such that $P=\phi\left(\left[g_{1}(a: b), g_{2}(a: b)\right]\right)$. Now $P=p_{12}$ if and 
only if

$$
\operatorname{det}\left[\begin{array}{cc}
g_{1}(a: b) & g_{2}(a: b) \\
a & b
\end{array}\right]=0 .
$$

Since this a homogeneous quadratic polynomial of two variables there must be 2 solutions up to multiplicity. Hence the coefficient of the monomial $x_{1} x_{2} x_{3} y_{12} y_{13} y_{23}$ is 2 .

The total number of terms in $A_{6} 141$ (this can be calculated by a standard inclusionexclusion argument). Since $\left|Z_{1}\right|=78,\left|Z_{2}\right|=62$, and $\left|Z_{3}\right|=1$ we have computed the coefficients of all possible terms. Finally with any computer software system one can expand the polynomial $\prod_{1 \leq i<j \leq 3}\left(x_{i}+y_{i j}\right)\left(x_{j}+y_{i j}\right)$ and see that the coefficients match what we have just determined. This completes the proof.

Remark 10. A generic element of $M$ can be thought of as a generic arrangement of 3 lines. The complement inside $M$ of this generic locus consists of several 5 dimensional subvarieties. These consist of arrangements where two lines are the same and arrangements consisting of a triple line, together with their marked points.

Now we determine the characteristic numbers for 3 lines.

Theorem 11. The characteristic numbers $N_{3}(p, 6-p)$ for a generic arrangement of 3 lines are given in the following table.

\begin{tabular}{|l||l|l|l|l|l|l|l|}
\hline$p$ & 0 & 1 & 2 & 3 & 4 & 5 & 6 \\
\hline$N_{3}(p, 6-p)$ & 15 & 30 & 48 & 57 & 48 & 30 & 15 \\
\hline
\end{tabular}

Proof. If we intersect $M$ with 6 generic conditions then we will obtain a finite number of points none of which will lie on the 5-dimensional non-generic subvarieties. So, when we multiply the class $[M]$ with that from Lemma 7 we will only be counting generic arrangements of 3 lines. From Lemma 9 we have

$$
[M]=\left[\cap_{1 \leq i<j \leq 3}\left(p_{i j} \in \ell_{i}\right) \cap\left(p_{i j} \in \ell_{j}\right)\right]=\prod_{1 \leq i<j \leq 3}\left(x_{i}+y_{i j}\right)\left(x_{j}+y_{i j}\right) .
$$

Then the condition that the arrangement $\mathcal{A}$ passes through a given point $p \in \mathbb{P}^{2}$ has class $x_{1}+x_{2}+x_{3}$. Similarly the condition that a given line $L \in \mathbb{P}^{2}$ contains one of the $p_{i j}$ has class $y_{12}+y_{13}+y_{23}$. Using the Moving Lemma, Lemma 7, and Remark8 the degree of the class $[M]\left(x_{1}+x_{2}+x_{3}\right)^{p}\left(y_{12}+y_{13}+y_{23}\right)^{6-p}$ in $A_{*}\left(\left(\mathbb{P}^{2 *}\right)^{3} \times\left(\mathbb{P}^{2}\right)^{3}\right)$ counts the number of labeled 3-generic arrangements that pass through $p$ general points and are tangent to $6-p$ general lines in $\mathbb{P}^{2}$. To remove the effect of the labeling, we divide these numbers by 3 ! to obtain the characteristic numbers. 
Remark 12. The symmetry of $N_{3}(p, 6-p)$ is easily explained: the dual of a 3-generic arrangement through $p$ points $P_{1}, \ldots, P_{p}$ and tangent to $6-p$ lines $L_{1}, \ldots, L_{6-p}$ is a 3generic arrangement through the $6-p$ dual points $\hat{L}_{1}, \ldots, \hat{L}_{6-p}$ and tangent to the $p$ dual lines $\hat{P}_{1}, \ldots, \hat{P}_{p}$. This symmetry does not occur in the 4 lines case as one can see below.

Now we consider the 4 line case. We compute the class $M$ as before but we use transversality arguments in place of the method of undetermined coefficients.

\section{Lemma 13. The set}

$$
M=\left\{\left(\ell_{1}, \ldots, \ell_{4}, p_{12}, \ldots, p_{34}\right) \in\left(\mathbb{P}^{2 *}\right)^{4} \times\left(\mathbb{P}^{2}\right)^{6}: p_{i j} \in \ell_{i} \cap \ell_{j} \text { for } 1 \leq i<j \leq 4\right\}
$$

is a quasi-projective variety of codimension 12 in $\left(\mathbb{P}^{2 *}\right)^{4} \times\left(\mathbb{P}^{2}\right)^{6}$. The Chow ring of the ambient space is

$$
A=A\left(\left(\mathbb{P}^{2 *}\right)^{4} \times\left(\mathbb{P}^{2}\right)^{6}\right) \cong \mathbb{Z}\left[x_{1}, \ldots, x_{4}, y_{12}, \ldots, y_{34}\right] /\left(x_{1}^{3}, \ldots, x_{4}^{3}, y_{12}^{3}, \ldots, y_{34}^{3}\right)
$$

where the $x_{i}$ correspond to the lines and the $y_{i j}$ correspond to the intersection points $p_{i j}$. In this ring the class of $M$ is

$$
[M]=\left[\cap_{1 \leq i<j \leq 4}\left(\left(p_{i j} \in \ell_{i}\right) \cap\left(p_{i j} \in \ell_{j}\right)\right)\right]=\prod_{1 \leq i<j \leq 4}\left(x_{i}+y_{i j}\right)\left(x_{j}+y_{i j}\right) .
$$

Proof. As in Lemma 9 set $\pi_{i}:\left(\mathbb{P}^{2 *}\right)^{4} \times\left(\mathbb{P}^{2}\right)^{6} \rightarrow \mathbb{P}^{2 *}$ to be the projection to the factor corresponding to the line $\ell_{i}$ and $\pi_{i j}:\left(\mathbb{P}^{2 *}\right)^{4} \times\left(\mathbb{P}^{2}\right)^{6} \rightarrow \mathbb{P}^{2}$ to be the projection to the factor corresponding to the point $p_{i j}$. The condition $p_{i j} \in \ell_{i}$ is a bilinear hypersurface in $\left(\mathbb{P}^{2 *}\right)^{4} \times\left(\mathbb{P}^{2}\right)^{6}$ whose class is $x_{i}+y_{i j}$. The closure of the variety $M$ is the intersection of these 12 hypersurfaces. We will show that this is a local complete intersection.

Put $\pi=\pi_{1} \times \pi_{2} \times \pi_{3} \times \pi_{4}$. Examine the restriction $\pi: M \rightarrow\left(\mathbb{P}^{2 *}\right)^{4}$. Now stratify $\left(\mathbb{P}^{2 *}\right)^{4}$ into the following quasi-projective subvarieties $G=$ "generic arrangements ", $T=$ "arrangements where two lines are equal and the other two are generic", $D=$ "arrangements where there are two generic double lines", $H=$ "arrangements where three lines are equal and the last one is generic", and $F=$ "arrangements where all four lines are equal". So, $\left(\mathbb{P}^{2 *}\right)^{4}=G \cup D \cup T \cup H \cup F$ and all the unions are disjoint. The dimension of $G$ is 8 and for any $x \in G$ the fiber $\pi^{-1}(x)$ is a unique point. Hence $\pi^{-1}(G)$ is 8 -dimensional. The dimension of $T$ is 6 and for any $x \in T$ the fiber $\pi^{-1}(x)$ is 1 -dimensional. Hence $\pi^{-1}(T)$ is 7-dimensional. The dimension of $D$ is 4 and for $x \in D$ the fiber $\pi^{-1}(x)$ is 2-dimensional. Hence $\pi^{-1}(D)$ is 6-dimensional. The dimension of $H$ is 4 and for $x \in H$ the fiber $\pi^{-1}(x)$ is 3-dimensional. Hence $\pi^{-1}(H)$ is 7-dimensional. The dimension of $F$ 
is 2 and for $x \in F$ the fiber $\pi^{-1}(x)$ is 6-dimensional. Hence $\pi^{-1}(F)$ is 8-dimensional. Since $M=\pi^{-1}(G) \cup \pi^{-1}(T) \cup \pi^{-1}(D) \cup \pi^{-1}(H) \cup \pi^{-1}(F)$ we have shown that $M$ is 8-dimensional.

Hence $M$ is a local complete intersection in $\left(\mathbb{P}^{2 *}\right)^{4} \times\left(\mathbb{P}^{2}\right)^{6}$. Using Theorem 5.10 in [4] the class of the intersection $[M]$ is the product of the classes $x_{i}+y_{i j}$.

Theorem 14. The characteristic numbers $N_{4}(p, 8-p)$ for a generic arrangement of 4 lines are given in the following table.

\begin{tabular}{|l||l|l|l|l|l|l|l|l|l|}
\hline$p$ & 0 & 1 & 2 & 3 & 4 & 5 & 6 & 7 & 8 \\
\hline$N_{4}(p, 8-p)$ & 16695 & 17955 & 13185 & 8190 & 4410 & 2070 & 855 & 315 & 105 \\
\hline
\end{tabular}

Proof. We use an argument similar to the proof of Theorem 11 but there is a subtlety. The component $F$ from Lemma 13 consisting of quadruple lines has the same dimension as the component $G$ of generic arrangements of 4 lines. It follows that $[M]=a[F]+b[G]$ for some integers $a$ and $b$. Specialize the equations that cut out $M$ to a fixed generic arrangement $\mathcal{A}$ of 4 lines to see that there is just one element of $M$ lying over $\mathcal{A}$, with multiplicity one; this shows that $a=1$. Similarly, if we specialize the equations that cut out $M$ to $\mathcal{A}$, a fixed quadruple line with six fixed marked points $p_{i j}$, we again see that the fiber of $M$ lying over $\mathcal{A}$ is a reduced point in $\mathcal{M}$; so $b=1$ and $[M]=[F]+[G]$. Let $C=\left(x_{1}+x_{2}+x_{3}+x_{4}\right)^{p}\left(y_{12}+y_{13}+y_{14}+y_{23}+y_{24}+y_{34}\right)^{8-p}$. The degree of $[M] C=[F] C+[G] C$ also counts some instances in which all the $\ell_{i}$ are equal (coming from the term $[F] C$ ). For instance, in the case where $p=0$, we seek elements in $M$ with some $p_{i j}$ on each of 8 lines $L_{1}, \ldots, L_{8}$. If we set all the $\ell_{i}$ equal to the line joining $L_{i} \cap L_{j}$ to $L_{s} \cap L_{t}$ then the common line $\ell_{i}$ meets the 8 lines $L_{j}$ in just $\left(\begin{array}{l}4 \\ 2\end{array}\right)=6$ points. Labeling these points with the six points $p_{i j}$ gives an element of $M$ that is included in our degree count but that doesn't correspond to a 4-generic arrangement; this is illustrated in the left picture of Figure 2, where the thick line represents the common line $\ell_{i}$. There are $\left(\begin{array}{c}8 \\ 2,2,4\end{array}\right) 6 ! / 2$ ways to produce such examples. Removing these from the degree count, we obtain the degree of $[G] C$ and dividing the result by 4 ! to account for the effect of labeling, we obtain the value of $N_{4}(0,8)$ in the Table.

Similarly, quadruple lines are counted in the degree computations for $p=1$ and $p=2$, as illustrated in the middle and right pictures of Figure 2. This requires an adjustment of $\left(\begin{array}{c}8 \\ 2,6\end{array}\right) 6$ ! in the case of $p=1$ and 6 ! in the case of $p=2$, giving rise to the values for $N_{4}(p, 8-p)$ in the statement of the theorem.

Remark 15. It would be interesting to have an explanation for the unimodality of the characteristic numbers $N_{k}(p, 2 k-p)$. In particular, do the characteristic numbers $\left\{N_{k}(p, 2 k-\right.$ $p)\}$ for generic arrangements of $k$ lines always form a unimodal sequence? 

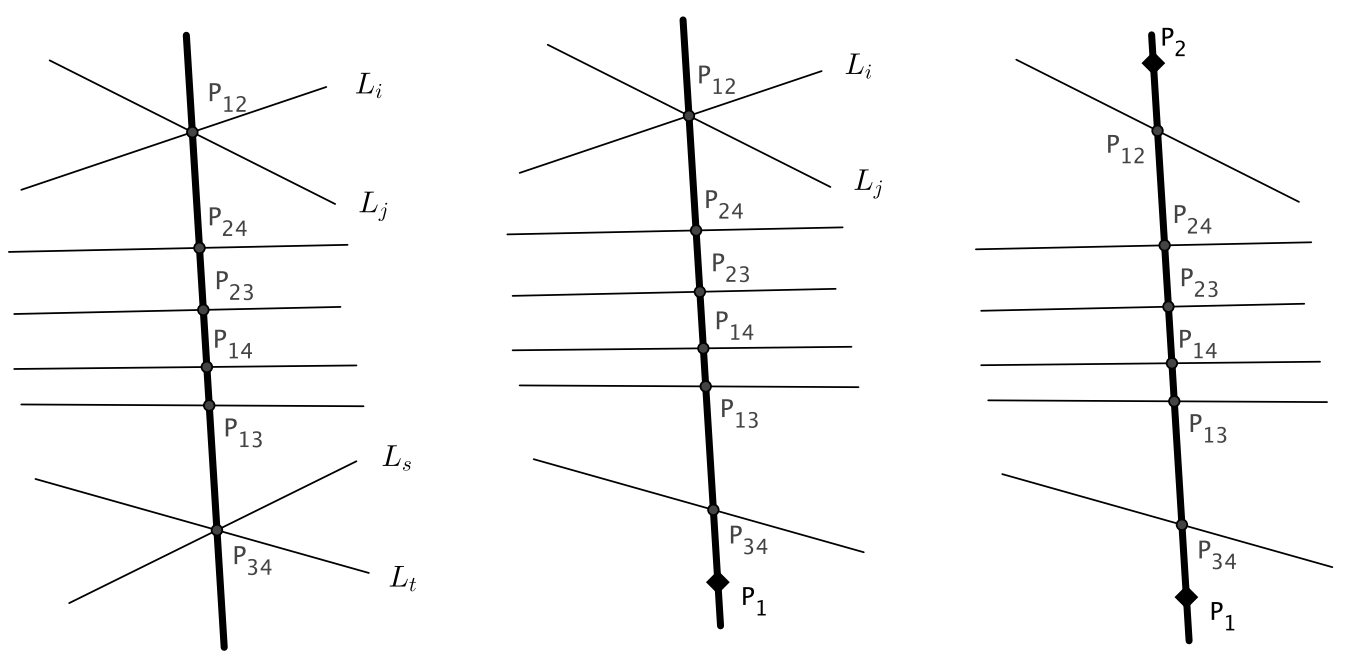

Figure 2: Quadruple lines counted by the degree computation for $p=0$ (left), $p=1$ (middle), $p=2$ (right).

Remark 16. Taking the dual of the 4-generic arrangement yields a braid line arrangement $\mathcal{A}_{3}$, an arrangement of 6 lines meeting in 4 triple points and 3 double points, pictured in Figure 3. This is related to the braid arrangement (the reflecting hyperplanes of the action of $\mathcal{S}_{4}$ on the variables of $\left.\mathbb{C}^{4}\right), \mathcal{A}_{3}=\left\{x_{i}=x_{j}: 1 \leq i<j \leq 4\right\}$ in $\mathbb{C}^{4}$ which consists of 6 hyperplanes, each containing the line $\left\{x_{1}=x_{2}=x_{3}=x_{4}\right\} \subset \mathbb{C}^{4}$. Quotienting out the common line and projectivizing gives the braid arrangement in $\mathbb{P}^{2}$.

The moduli space $\mathcal{M}_{\mathcal{A}_{3}}$ has the same dimension, 8 , as the moduli space of the dual of $\mathcal{A}_{3}$. The characteristic numbers $N_{\mathcal{A}_{3}}(p, 8-p)$ counting the number of arrangements combinatorially equivalent to the braid arrangement that pass through $p$ points and are tangent to $8-p$ lines in general position are given by $N_{4}(8-p, p)$. For example, there are 16695 braid arrangements through 8 points in general position in $\mathbb{P}^{2}$, a result initially reported in Paul [20].

The characteristic numbers are important because they determine the answer to all enumerative questions involving points and tangency to arbitrary smooth curves (see [7, section $10.4]$ and the references therein for the history of this result).

Theorem 17. Let $\mathcal{L}_{\mathcal{A}}$ be the intersection lattice of a line arrangement and let $t=\operatorname{dim} \mathcal{M}_{\mathcal{A}}$. The number of line arrangements with intersection lattice isomorphic to $\mathcal{L}_{\mathcal{A}}$ through $p$ 


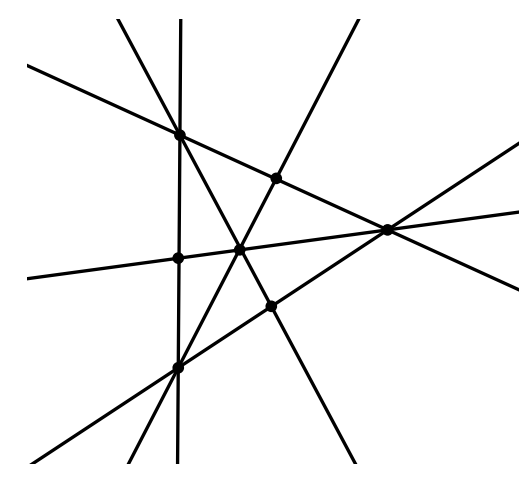

Figure 3: The braid arrangement $\mathcal{A}_{3}$

points and tangent to $t-p$ smooth curves of degrees $n_{1}, \ldots, n_{t-p}$ and classes ${ }^{1} m_{1}, \ldots, m_{t-p}$ in general position is obtained from the product

$$
\mu^{p} \prod_{i=1}^{t-p}\left(m_{i} \mu+n_{i} \nu\right)
$$

by expanding the polynomial and replacing the monomial $\mu^{k} \nu^{t-k}$ by the associated characteristic number - the number of arrangements with lattice type $\mathcal{L}_{\mathcal{A}}$ passing through $k$ general points and tangent to $t-k$ lines.

Proof. The proof follows immediately from the argument due to Fulton and MacPherson in [7, section 10.4] if we interpret each point condition as a tangency condition to a curve of degree 0 and class 1 . Alternatively, we can work with the dimension $(t-p)$ family of arrangements in $\mathcal{M}_{\mathcal{A}}$ that pass through the specified $p$ points, apply Fulton and MacPherson's theorem and then re-interpret the results in terms of the polynomial displayed above.

Example 18. To compute the number of generic arrangements of 4 lines through 3 points, tangent to a given line and tangent to four smooth conics, we expand the product $\mu^{3}(2 \mu+$ $2 \nu)^{4} \nu^{1}$ and replace the monomials with the appropriate quantities from Theorem 14 . The answer is

$$
2^{4}(315)+2^{4}\left(\begin{array}{l}
4 \\
1
\end{array}\right)(855)+2^{4}\left(\begin{array}{l}
4 \\
2
\end{array}\right)(2070)+2^{4}\left(\begin{array}{l}
4 \\
3
\end{array}\right)(4410)+2^{4}(8190)=671760 .
$$

\footnotetext{
${ }^{1}$ The class of a curve is the number of lines passing through a given general point and tangent to the curve at a simple point. For example, the class of a smooth curve of degree $d$ is $d(d-1)$.
} 
Remark 19. The computation of $N_{k}(p, 2 k-p)$ becomes more intricate as $k$ increases. For instance, using the method of proof from Theorem 14 to compute $N_{5}(0,10)$, we see that every quintuple line determines a point in the incidence correspondence $M$. This higher-dimensional component of the solution space (isomorphic to $\mathbb{P}^{2}$ ) "counts" as a finite number of points in the standard intersection theory computation of $N_{5}(0,10)$. The appropriate count is called the excess intersection of this component and can be computed using the Excess Intersection Formula [7, section 6.3]. We do not pursue this approach further here.

\section{Pencils and Cones Over Generic Arrangements}

An arrangement of lines is said to be a pencil if all lines pass through a common point. Such an arrangement is also a 0 -coned generic arrangement. We start this section with a simple enumerative result about pencils.

Theorem 20. If $\mathcal{A}$ is a pencil of $k$ lines in $\mathbb{P}^{2}$ then $\operatorname{dim} \mathcal{M}_{\mathcal{A}}=k+2$ and the characteristic numbers are $N_{\mathcal{A}}(k+2,0)=3\left(\begin{array}{c}k+2 \\ 4\end{array}\right), N_{\mathcal{A}}(k+1,1)=\left(\begin{array}{c}k+1 \\ 2\end{array}\right), N_{\mathcal{A}}(k, 2)=1$. All other characteristic numbers are 0.

Proof. By the Pigeonhole Principle, if a pencil $\mathcal{A}$ of $k$ lines passes through more than $k+3$ points in general position then 3 of the lines must each contain 2 points and all three lines must be coincident, but this means that these 6 points must satisfy an algebraic relation and thus violates the assumption that the points were in general position. As well, it is easy to see that there are a finite number of pencils $\mathcal{A}$ of $k$ lines passing through $k+2$ points in general position (two lines contain 2 points each and intersect in the node of the pencil, the other $k-2$ lines each contain one of the remaining points) so $\operatorname{dim} \mathcal{M}_{\mathcal{A}}=k+2$. There are $N_{\mathcal{A}}(k+2,0)=\left(\begin{array}{c}k+2 \\ 2,2, k-2\end{array}\right) / 2=3\left(\begin{array}{c}k+2 \\ 4\end{array}\right)$ ways to create such an arrangement.

To compute $N_{\mathcal{A}}(k+1,1)$ note that the node of the pencil must lie on the given line. The arrangement must have one line containing 2 of the $k+1$ points and this line intersects the given line at the node of the pencil; the remaining $k-1$ lines in the pencil each contain 1 point and the node. There are $N_{\mathcal{A}}(k+1,1)=\left(\begin{array}{c}k+1 \\ 2\end{array}\right)$ ways to choose the first line in the pencil; the rest of the construction is determined. Finally, to compute $N_{\mathcal{A}}(k, 2)$ note that the node must lie at the intersection of the two given lines and that each line in the pencil must pass through the node and one of the $k$ given points. So $\mathcal{A}$ is uniquely determined.

We proceed to study $d$-coned generic arrangements with $d \geq 1$. Recall from Definition 2 that an arrangement $\mathcal{A}$ of $k>n$ hyperplanes in $\mathbb{P}^{n}$ is a $d$-coned generic arrangement if $\mathcal{A}$ can be obtained from a generic hyperplane arrangement $\mathcal{B}$ in a linear subspace $H \cong \mathbb{P}^{n-d-1}$ 
by taking the cone with a $d$-dimensional linear space (equivalently, with $d+1$ general points). Given a $d$-coned generic arrangement $\mathcal{A}$ in $\mathbb{P}^{n}$, the intersection with a general linear space $H$ of dimension $n-d-1$ is a generic hyperplane arrangement in $\mathbb{P}^{n-d-1}$.

Lemma 21. Let $\mathcal{A}$ be a d-coned generic arrangement of $k$ hyperplanes in $\mathbb{P}^{n}$. Then the dimension of the moduli space $\mathcal{M}_{\mathcal{A}}$ is $D=(d+1)(n-d)+k(n-d-1)$.

Proof. Note that an arrangement $\mathcal{B} \in \mathcal{M}_{\mathcal{A}}$ is determined by $\Lambda$ in the Grassmannian $\mathbb{G}(d, n)$ of $d$-dimensional linear spaces of $\mathbb{P}^{n}$, together with $k$ points in $\mathbb{P}\left(\mathbb{C}^{n+1} / \Lambda\right) \cong \mathbb{P}^{n-d-1}$ (since $\Lambda$ determines a subspace of dimension $d+1$ in $\mathbb{C}^{n+1}$ ). So the dimension of $\mathcal{M}_{\mathcal{A}}$ is $D=\operatorname{dim}\left[\mathbb{G}(d, n) \times\left(\mathbb{P}^{n-d-1}\right)^{k}\right]=(d+1)(n-d)+k(n-d-1)$.

As a warm-up, we count the number of 0 -coned generic arrangements through a set of points.

Theorem 22. Let $\mathcal{A}$ be a 0 -coned generic arrangement of $k \geq n$ hyperplanes in $\mathbb{P}^{n}$. Then the number of arrangements combinatorially equivalent to $\mathcal{A}$ that pass through $k n+n-k$ points in general position is

$$
N_{\mathcal{A}}=\frac{1}{k !}\left(\begin{array}{l}
k \\
n
\end{array}\right)\left(\begin{array}{c}
k n+n-k \\
(n)^{n},(n-1)^{k-n}
\end{array}\right)=\frac{(k n+n-k) !}{(n !)^{n+1}((k-n) !)((n-1) !)^{k-n}} .
$$

Proof. By Lemma 21, $\operatorname{dim} \mathcal{M}_{\mathcal{A}}=k n+n-k$. The only way to obtain an arrangement combinatorially equivalent to $\mathcal{A}$ through the $k n+n-k$ points is to have $n$ hyperplanes pass through $n$ of the points and meet in a new point $p$; then each of the remaining $k-$ $n$ of the hyperplanes pass through the point $p$ and $n-1$ of the remaining given points. Labeling the set of hyperplanes with $n$ points as $H_{1}, \ldots, H_{n}$ and the remaining hyperplanes $H_{n+1}, \ldots, H_{k}$, we have $\left(\begin{array}{c}k n+n-k \\ (n)^{n},(n-1)^{k-n}\end{array}\right)$ labeled arrangements. Dividing by $n ! k-n$ ! gives the number of unlabeled arrangements and rearranging terms gives the desired formula.

It is tempting to think that the number of $d$-coned generic arrangements of $k$ hyperplanes in $\mathbb{P}^{n}$ that pass through $D=(d+1)(n-d)+k(n-d-1)$ points in general position is just

$$
N_{\mathcal{A}}=\frac{1}{k !}\left(\begin{array}{l}
k \\
n
\end{array}\right)\left(\begin{array}{c}
D \\
(n)^{n-d},(n-d)^{k-n+d}
\end{array}\right),
$$

but in fact this grossly undercounts the arrangements. In this sense, the 0-coned generic arrangement result in Theorem 22 is misleading.

Before stating our result on the enumeration of $d$-coned generic arrangements, we quickly review the structure of the intersection ring $A=A(\mathbb{G}(d, n))$ of the Grassmannian $\mathbb{G}(d, n)$ of $d$-planes in $\mathbb{P}^{n}$. Given any complete flag of linear spaces $F: F_{0} \subsetneq F_{1} \subsetneq \ldots \subsetneq$ 
$F_{n}=\mathbb{P}^{n}$ with $\operatorname{dim} F_{j}=j$, we have a cellular decomposition of $\mathbb{G}(d, n)$ into affine cells; for each $(d+1)$-tuple $\left(\alpha_{0}, \alpha_{1}, \ldots, \alpha_{d}\right)$ of non-increasing non-negative integers $\alpha_{i} \leq n-d$ we have an affine cell $X_{\alpha}(F)$ of codimension $|\alpha|=\sum \alpha_{i}$. To describe the $d$-planes $\Lambda$ in $X_{\alpha}(F)$, note that for each $d$-plane $\Lambda$ the sequence $\operatorname{dim} \Lambda \cap F_{j}$ is completely characterized by the $d+1$ places where the dimension increases, the so-called rank jumps. Now $\Lambda \in X_{\alpha}(F)$ precisely when the collection of rank jumps is $\left\{n-d+j-\alpha_{j}: 0 \leq j \leq d\right\}$. For instance the largest open cell $X_{(0){ }^{d+1}}$ consists of $d$-planes in general position with respect to the flag $F$. See Hatcher [11] for more details on the cellular decomposition of $\mathbb{G}(d, n)$. Define the intersection class $\sigma_{\alpha} \in A$ to be the class of the closure of $X_{\alpha}(F)$. The set of $\sigma_{\alpha}$ form an additive basis for the intersection ring $A(\mathbb{G}(d, n))$. The multiplicative structure of the intersection ring is described by the Pieri and Giambelli formulas (see e.g. Gatto [8,9] for statements of these results and an interesting description of the multiplicative structure of $A(\mathbb{G}(d, n))$ in terms of Hasse-Schmidt derivations). The class $\sigma_{(0)} d+1$ is the multiplicative identity in $A(\mathbb{G}(d, n))$. We denote the tuple consisting of $i$ 1's followed by $d+1-i 0$ 's by $1^{i}$. Similarly, the tuple consisting of $d+1$ copies of $n-d$ is denoted $(n-d)^{d+1}$. The class $\sigma_{(n-d)^{d+1}}$ is the class of a point and the class $\sigma_{1^{d+1}}$ is the class of the set of $d$-planes contained in $F_{n-1} \cong \mathbb{P}^{n-1}$. Though we will not need the full power of the Pieri and Giambelli formulas, we note that there is a duality between the classes $\sigma_{\alpha}$ in the sense that

$$
\sigma_{\alpha} \cdot \sigma_{r\left((n-d)^{d+1}-\alpha\right)}=\sigma_{(n-d)^{d+1}},
$$

where $r$ is the operation that reverses a tuple: $r\left(\beta_{0}, \ldots, \beta_{d}\right)=\left(\beta_{d}, \ldots, \beta_{0}\right)$. As well, if $\sigma_{\alpha_{1}}, \ldots, \sigma_{\alpha_{t}}$ are intersection classes with $\alpha_{1}+\cdots+\alpha_{t}=\operatorname{dim} \mathbb{G}(d, n)=(n-d)(d+$ 1) then their product has a well-defined degree, denoted $\int_{\mathbb{G}(d, n)} \sigma_{\alpha_{1}} \cdots \sigma_{\alpha_{t}}$, which can be interpreted as the number of $d$-planes in $X_{\alpha_{1}} \cap \cdots \cap X_{\alpha_{t}}$ if the classes $X_{\alpha_{i}}$ meet transversally (here the $X_{\alpha_{i}}$ can be interpreted as coming from different flags, in general position with respect to each other).

Theorem 23. If $\mathcal{A}$ is a d-coned generic arrangement of $k$ hyperplanes in $\mathbb{P}^{n}$, then the number of pencils combinatorially equivalent to $\mathcal{A}$ that pass through $D=(d+1)(n-d)+$ $k(n-d-1)$ points in general position is given by

$$
N_{\mathcal{A}}=\frac{1}{k !} \sum_{\Gamma} \sigma^{s}\left(\begin{array}{c}
k \\
s_{0}, s_{1}, \ldots, s_{d+1}
\end{array}\right)\left(\begin{array}{c}
D \\
(n-(d+1))^{s_{0}},(n-d)^{s_{1}}, \ldots,(n)^{s_{d+1}}
\end{array}\right),
$$

where

$$
\Gamma=\left\{\left(s_{0}, \ldots, s_{d+1}\right) \in \mathbb{N}^{d+2}: \sum_{i=0}^{d+1} i s_{i}=(d+1)(n-d)=\operatorname{dim} \mathbb{G}(d, n), \sum_{i=0}^{d+1} s_{i}=k\right\}
$$


and

$$
\sigma^{s}=\left(\sigma_{0}\right)^{s_{0}}\left(\sigma_{1}\right)^{s_{1}}\left(\sigma_{(1)^{2}}\right)^{s_{2}} \cdots\left(\sigma_{(1)^{d+1}}\right)^{s_{d+1}},
$$

using the notation of Remark 3 .

Proof. We first note that the intersection ring $A\left(\mathbb{G}(d, n) \times\left(\mathbb{P}^{n *}\right)^{k}\right)$ is just the tensor product of $A(\mathbb{G}(d, n))$ with $A\left(\left(\mathbb{P}^{n *}\right)^{k}\right)$. Now we determine the class $[Z] \in A\left(\mathbb{G}(d, n) \times \mathbb{P}^{n *}\right)$, where $Z$ is the closed set determining an incidence correspondence,

$$
Z=\left\{(\Lambda, H) \in \mathbb{G}(d, n) \times \mathbb{P}^{n *}: \Lambda \subset H\right\} .
$$

Note that $\mathbb{G}(d, n) \times \mathbb{P}^{n *}$ has dimension $(d+1)(n-d)+n$ and $Z$ has dimension $(d+1)(n-$ $d)+(n-d-1)$ so $Z$ has codimension $d+1$. It follows that

$$
[Z]=\sum_{\{\alpha:|\alpha| \leq d+1\}} a_{\alpha} \sigma_{\alpha} h^{d+1-|\alpha|},
$$

where $\sigma_{\alpha}$ and $h$ denote the pullbacks of these classes to $A\left(\mathbb{G}(d, n) \times \mathbb{P}^{n *}\right)$ and the $a_{\alpha}$ are integers.

We multiply $[Z]$ by $\sigma_{r\left((n-d)^{d+1}-\alpha\right)} h^{n-d-1+|\alpha|}$ to obtain $a_{\alpha} \sigma_{(n-d)^{d+1}} h^{n}$, representing $a_{\alpha}$ elements $(\Lambda, H) \in Z$ that also meet the flag $F$ in a manner prescribed by the class $\sigma_{r\left((n-d)^{d+1}-\alpha\right)} h^{n-d-1+|\alpha|}$. That is, $\Lambda$ has rank jumps at positions $n-d-\left(n-d-\alpha_{d}\right)=$ $\alpha_{d}, 1+\alpha_{d-1}, \ldots, d+\alpha_{0}$ (or earlier) and we can assume that $H$ must pass through $n-d-$ $1+|\alpha|$ points in general position.

Since $|\alpha| \leq d+1$ and $\alpha \in \mathbb{N}^{d+1}$, we see that if any $\alpha_{i}>1$ then there are at least $d-|\alpha|+2$ trailing zeros in $\alpha$. It follows that the first set of rank jumps occur at positions $0,1,2, \ldots, d-|\alpha|+1$. Now $H \supset \Lambda \supset F_{d-|\alpha|+1}$, which forces $H$ to pass through $d-|\alpha|+2$ points in general position in $F_{d-|\alpha|+1}$. Together with the previous $n-d-1+|\alpha|$ points in $H$, this forces $H$ to pass through $n+1$ points in general position in $\mathbb{P}^{n}$. Of course this is not possible, so $a_{\alpha}=0$ if any entry of $\alpha$ is greater than 1 .

On the other hand, if $\alpha=(1)^{d+1-\ell}$ then we multiply [Z] by $\sigma_{(n-d)^{\ell}(n-d-1)^{d+1-\ell}} h^{n-\ell}$ to get $a_{(1)^{d+1-\ell}} \sigma_{(n-d)^{d+1}} h^{n}$, representing $a_{(1)^{d+1-\ell}}$ elements $(\Lambda, H) \in Z$ such that $\Lambda$ has rank jumps at positions in $0,1, \ldots, \ell-1, \ell+1, \ldots, d+1$ (or earlier) and $H$ contains $n-\ell$ points in general position. But then $H \supset \Lambda \supset F_{\ell-1}$ and together with the conditions imposed by the $n-\ell$ points, $H$ is uniquely determined. Then $\Lambda=H \cap F_{d+1}$ is also uniquely determined so $a_{\alpha}=a_{(1)^{d+1-\ell}}=1$ (in the case $\ell=d+1$, i.e. $\alpha=(0)^{d+1}, \Lambda=F_{d}$ and $H$ is uniquely determined by $H \supset \Lambda=F_{d}$ and the point conditions). It follows that the class $[Z] \in A\left(\mathbb{G}(d, n) \times \mathbb{P}^{n *}\right)$ is

$$
[Z]=\sum_{\ell=0}^{d+1} \sigma_{(1)^{d+1-\ell}} h^{\ell} .
$$


Now we return to our main result and determine the number of hyperplane arrangements consisting of $k$ hyperplanes in $\mathbb{P}^{n}$ that all contain a $d$-dimensional linear space and pass through $D$ points in general position. Let

$$
Z_{i}=\left\{\left(\Lambda, H_{1}, \ldots, H_{k}\right) \in \mathbb{G}(d, n) \times\left(\mathbb{P}^{n *}\right)^{k}: \Lambda \subset H_{i}\right\}
$$

and write $h_{i}$ and $\sigma_{\alpha}$ for the pull-backs of the obvious classes to $\mathbb{G}(d, k) \times\left(\mathbb{P}^{n *}\right)^{k}$. Note that $\left[Z_{i}\right]=\sum_{\ell=0}^{d+1} \sigma_{(1)^{d+1-\ell}} h_{i}^{\ell} \in A\left(\mathbb{G}(d, n) \times\left(\mathbb{P}^{n *}\right)^{k}\right)$ and $Z_{i}$ is a local complete intersection.

Now we show that $\bigcap_{i=1}^{k} Z_{i}$ is a local complete intersection. The dimension of the ambient space is $\operatorname{dim} \mathbb{G}(d, n)+n k$ and the dimension of $\bigcap_{i=1}^{k} Z_{i}$ is $\operatorname{dim} \mathbb{G}(d, n)+(n-(d+$ 1)) $k$, showing that $\bigcap_{i=1}^{k} Z_{i}$ has codimension $k(d+1)$. Since each $Z_{i}$ has codimension $d+1$ and is a local complete intersection, their intersection is also a local complete intersection. Again using Theorem 5.10 of [4], we have that on the level of classes

$$
\left[\bigcap_{i=1}^{k} Z_{i}\right]=\left[Z_{1}\right]\left[Z_{2}\right] \cdots\left[Z_{k}\right] .
$$

Let $P_{1}, \ldots, P_{D}$ be points in general position in $\mathbb{P}^{n}$ and for each $P_{i}$ let

$$
Y_{i}=\left\{\left(\Lambda, H_{1}, \ldots, H_{k}\right) \in \mathbb{G}(d, n) \times\left(\mathbb{P}^{n *}\right)^{k}: P_{i} \in H_{1} \cup \cdots \cup H_{k}\right\} .
$$

Note that $\left[Y_{i}\right]=h_{1}+\cdots+h_{k}$. Again from Lemma 7 and using Kleiman's Transversality Theorem (see Kleiman's fundamental paper [14]) we have that at the level of classes

$$
\left[\left(\cap_{i=1}^{k} Z_{i}\right) \cap\left(\cap_{j=1}^{D} Y_{j}\right)\right]=\left[\left(\cap_{i=1}^{k} Z_{i}\right)\right]\left[\left(\cap_{j=1}^{D} Y_{j}\right)\right]=\left[Z_{1}\right]\left[Z_{2}\right] \cdots\left[Z_{k}\right]\left[Y_{1}\right]\left[Y_{2}\right] \cdots\left[Y_{D}\right] .
$$

Now

$$
\begin{aligned}
& {\left[\left(\cap_{i=1}^{k} Z_{i}\right) \cap\left(\cap_{j=1}^{D} Y_{j}\right)\right]=\left[Z_{1}\right] \cdots\left[Z_{k}\right]\left[Y_{1}\right] \cdots\left[Y_{D}\right]} \\
& =\left[\prod_{i=1}^{k}\left(\sum_{\ell=0}^{d+1} \sigma_{(1)^{d+1-\ell}} h_{i}^{\ell}\right)\right]\left(h_{1}+\cdots+h_{k}\right)^{D} \\
& \left.=\sum_{\Gamma} \sigma^{s}\left(\begin{array}{c}
k \\
s_{0}, s_{1}, \ldots, s_{d+1}
\end{array}\right){ }_{(n-(d+1))^{s_{0}},(n-d)^{s_{1}, \ldots,},(n)^{s_{d+1}}}\right) \text {, }
\end{aligned}
$$

where $\Gamma=\left\{\left(s_{0}, \ldots, s_{d+1}\right) \in \mathbb{N}^{d+2}: \sum_{i=0}^{d+1} i s_{i}=(d+1)(n-d)=\operatorname{dim} \mathbb{G}(d, n), \sum_{i=0}^{d+1} s_{i}=\right.$ $k\}$ and

$$
\sigma^{s}=\left(\sigma_{0}\right)^{s_{0}}\left(\sigma_{1}\right)^{s_{1}}\left(\sigma_{(1)^{2}}\right)^{s_{2}} \cdots\left(\sigma_{(1)^{d+1}}\right)^{s_{d+1}} .
$$

Since each unordered arrangement of hyperplanes gives $k$ ! arrangements of labeled hyperplanes, we divide by $k$ ! to obtain the correct degree $N_{\mathcal{A}}$. Since the points $P_{i}$ are in general position, Kleiman's transversality theorem assures us that $\left(\cap_{i=1}^{k} Z_{i}\right) \cap\left(\cap_{j=1}^{D} Y_{j}\right)$ is a finite collection of points, each appearing with multiplicity one. So we can interpret the above computation as saying that there are $N_{\mathcal{A}} d$-coned generic arrangements of $k$ hyperplanes in $\mathbb{P}^{n}$ that pass through $D$ points in general position. 
It would be interesting to study the enumerative geometry of hyperplane arrangements in a combinatorial equivalence class different from the generic arrangements and $d$-coned generic arrangements. In general this seems to require some subtle intersection theory computations. In particular, it would be interesting to see if intersection theory computations on the moduli space of stable maps can deal with the enumerative geometry of more general families of line arrangements.

Remark 24. The result in Theorem 23 takes a particularly nice shape when $d=1$. In this case $\sigma^{s}=\left(\sigma_{0}\right)^{s_{0}}\left(\sigma_{1}\right)^{s_{1}}\left(\sigma_{11}\right)^{s_{2}}$ counts the number of lines that are in $s_{2}$ general hyperplanes in $\mathbb{P}^{n}$ and meet $s_{1}$ general codimension-2 planes. Cutting down by the hyperplanes, this is the number of lines in $\mathbb{P}^{n-s_{2}}$ that meet $s_{1}=2(n-1)-2 s_{2}$ codimension- 2 planes. This was one of the classical problems studied by Schubert [22], who showed that $\sigma^{s}=C_{n-s_{2}}$, where $C_{N}=\frac{1}{N}\left(\begin{array}{c}2 N-2 \\ N-1\end{array}\right)$ defines the sequence of Catalan numbers (with the index shifted to start at 1$)$.

Example 25. The number of 1-coned generic arrangements of 9 hyperplanes in $\mathbb{P}^{5}$ that go through 35 points is

$$
\begin{aligned}
& N_{\mathcal{A}}=\frac{1}{9 !}\left[\left(\begin{array}{c}
9 \\
5,0,4
\end{array}\right)\left(\begin{array}{c}
35 \\
(3)^{5}(4)^{0}(5)^{4}
\end{array}\right)+\left(\begin{array}{c}
9 \\
4,2,3
\end{array}\right)\left(\begin{array}{c}
35 \\
(3)^{4}(4)^{2}(5)^{3}
\end{array}\right)+2\left(\begin{array}{c}
9 \\
3,4,2
\end{array}\right)\left(\begin{array}{c}
35 \\
(3)^{3}(4)^{4}(5)^{2}
\end{array}\right)+\right. \\
& \left.5\left(\begin{array}{c}
9 \\
2,6,1
\end{array}\right)\left(\begin{array}{c}
35 \\
(3)^{2}(4)^{6}(5)^{1}
\end{array}\right)+14\left(\begin{array}{c}
9 \\
1,8,0
\end{array}\right)\left(\begin{array}{c}
35 \\
(3)^{1}(4)^{8}(5)^{0}
\end{array}\right)\right] \\
& =148,467,792,706,702,950,173,442,750 \text {. }
\end{aligned}
$$

\section{References}

[1] Paolo Aluffi. The enumerative geometry of plane cubics. I. Smooth cubics. Trans. Amer. Math. Soc., 317(2):501-539, 1990.

[2] Federico Ardila. Computing the Tutte polynomial of a hyperplane arrangement. $\mathrm{Pa}$ cific J. Math., 230(1):1-26, 2007.

[3] Enrico Carlini. Codimension one decompositions and Chow varieties. In Projective varieties with unexpected properties, pages 67-79. Walter de Gruyter GmbH \& Co. KG, Berlin, 2005.

[4] David Eisenbud and Joe Harris. 3264 and all that. Available at http://isites.harvard.edu/fs/docs/icb.topic720403.files/book.pdf.

[5] László M. Fehér, András Némethi, and Richárd Rimányi. Equivariant classes of matrix matroid varieties. Comment. Math. Helv., 87(4):861-889, 2012. 
[6] Alex Fink and David E. Speyer. $K$-classes for matroids and equivariant localization. Duke Math. J., 161(14):2699-2723, 2012.

[7] William Fulton. Intersection theory, volume 2 of Ergebnisse der Mathematik und ihrer Grenzgebiete (3) [Results in Mathematics and Related Areas (3)]. SpringerVerlag, Berlin, 1984.

[8] Letterio Gatto. Schubert calculus: an algebraic introduction. Publicações Matemáticas do IMPA. [IMPA Mathematical Publications]. Instituto Nacional de Matemática Pura e Aplicada (IMPA), Rio de Janeiro, 2005. 25o Colóquio Brasileiro de Matemática. [25th Brazilian Mathematics Colloquium].

[9] Letterio Gatto. Schubert calculus via Hasse-Schmidt derivations. Asian J. Math., 9(3):315-321, 2005.

[10] Paul Hacking, Sean Keel, and Jenia Tevelev. Compactification of the moduli space of hyperplane arrangements. J. Algebraic Geom., 15(4):657-680, 2006.

[11] Allen Hatcher. Vector bundles and K-theory. Available online at http://www.math. cornell.edu/ hatcher/VBKT/VBpage.html.

[12] Mikhail M. Kapranov. Chow quotients of Grassmannians. I. In I. M. Gel'fand Seminar, volume 16 of Adv. Soviet Math., pages 29-110. Amer. Math. Soc., Providence, RI, 1993.

[13] Sheldon Katz. Enumerative geometry and string theory, volume 32 of Student Mathematical Library. American Mathematical Society, Providence, RI, 2006. IAS/Park City Mathematical Subseries.

[14] Steven L. Kleiman. The transversality of a general translate. Compositio Math., 28:287-297, 1974.

[15] Steven L. Kleiman. Problem 15: rigorous foundation of Schubert's enumerative calculus. In Mathematical developments arising from Hilbert problems (Proc. Sympos. Pure Math., Northern Illinois Univ., De Kalb, Ill., 1974), pages 445-482. Proc. Sympos. Pure Math., Vol. XXVIII. Amer. Math. Soc., Providence, R. I., 1976.

[16] Steven L. Kleiman and Robert Speiser. Enumerative geometry of nonsingular plane cubics. In Algebraic geometry: Sundance 1988, volume 116 of Contemp. Math., pages 85-113. Amer. Math. Soc., Providence, RI, 1991. 
[17] Maxim Kontsevich and Yuri Manin. Gromov-Witten classes, quantum cohomology, and enumerative geometry. Comm. Math. Phys., 164(3):525-562, 1994.

[18] Nikolai E. Mnëv. The universality theorems on the classification problem of configuration varieties and convex polytopes varieties. In Topology and geometry-Rohlin Seminar, volume 1346 of Lecture Notes in Math., pages 527-543. Springer, Berlin, 1988.

[19] Peter Orlik and Hiroaki Terao. Arrangements of hyperplanes, volume 300 of Grundlehren der Mathematischen Wissenschaften [Fundamental Principles of Mathematical Sciences]. Springer-Verlag, Berlin, 1992.

[20] Thomas Paul. The enumerative geometry of hyperplane arrangements. Trident Project, U.S. Naval Academy, 2012.

[21] Qingchun Ren, Jürgen Richter-Gebert, and Bernd Sturmfels. Cayley-Bacharach Formulas. Preprint: ArXiv:1405.6438.

[22] Hermann Schubert. Die $n$-dimensionalen Verallgemeinerungen der fundamentalen Anzahlen unseres Raums. Math. Ann., 26(1):26-51, 1886.

[23] Alan D. Sokal. The multivariate Tutte polynomial (alias Potts model) for graphs and matroids. In Surveys in combinatorics 2005, volume 327 of London Math. Soc. Lecture Note Ser, pages 173-226. Cambridge Univ. Press, Cambridge, 2005.

[24] David E. Speyer. A matroid invariant via the $K$-theory of the Grassmannian. Adv. Math., 221(3):882-913, 2009.

[25] Hiroaki Terao. Moduli space of combinatorially equivalent arrangements of hyperplanes and logarithmic Gauss-Manin connections. Topology Appl., 118(1-2):255274, 2002. Arrangements in Boston: a Conference on Hyperplane Arrangements (1999).

[26] Ravi Vakil. The characteristic numbers of quartic plane curves. Canad. J. Math., 51(5):1089-1120, 1999.

[27] Ravi Vakil. Murphy's law in algebraic geometry: badly-behaved deformation spaces. Invent. Math., 164(3):569-590, 2006.

[28] Sergey Yuzvinsky. Free and locally free arrangements with a given intersection lattice. Proc. Amer. Math. Soc., 118(3):745-752, 1993. 
[29] Hieronymous G. Zeuthen. Almindelige egenskaber ved systemer af plane kurver. Kongelige Danske Vidensk- abernes Selskabs Skrifter Naturvidenskabelig og Mathematisk, 1873.

[30] Hieronymous G. Zeuthen. Lehrbuch der abzählenden Methoden der Geometrie. Teubner, Leipzig, 1914. 